\title{
An ordinary life: People with dementia living in a residential setting.
}

Abstract

The aim of this research was to explore the experiences of care support workers and family members of the impact of a new care approach in a specialised unit as it shifted from a clinical to an inclusive model, focused on creating an ordinary life for people with dementia and their families. The research was a partnership between the unit staff and university researchers. Using a qualitative approach, data were collected in focus groups with 11 family members and 9 staff members. Thematic analysis identified the themes personalised care for people with dementia, family involvement, and staff competence and confidence to care. A personalised approach to supporting people with dementia was considered paramount, communicative family - staff relationships enhanced the social environment, and competence enhanced confidence and quality care. Participants identified positive ways of working that benefited staff and families and they reported increased wellbeing for the people with dementia on the unit. Developing well-articulated and systematically implemented local models of care provide opportunities for family and staff creativity and engagement, enhancing care for people with dementia. Strong and effective leadership is required to enable these approaches to become a reality.

\section{Keywords}

Dementia, specialised care, participation, family involvement, health and social care professionals

\section{An Ordinary Life}

In an ordinary life, people exercise choice about where they live, who they associate with and what they do each day. The impairments associated with the progression of dementia can diminish these choices, making the ordinariness of everyday life unreachable, so that people with dementia are dependent on others to facilitate everyday life. 
Achieving an ordinary life is challenging in residential care. People with dementia may require care in specialised residential units because of safety concerns necessitating a more secure environment. Common difficulties faced by people with dementia include mood disturbances, issues with sleep, inability to maintain independence and rarely, psychological issues such as hallucinations (Savva et al. 2009). Support must be carefully crafted to achieve good care and quality of life. A culture that values and adopts person centred care requires ongoing attention and resourcing in a milieu that is awkwardly poised between the dual purposes of the resident's home and a health care setting (Vladeck, 2003). Contemporary approaches to supporting people with dementia emphasise that person centred care is provided as a minimum requirement (Brownie \& Nancarrow, 2013; Edvardsson, Fetherstonhaugh, \& Nay, 2010), relationship based care and social citizenship are also referred to as aims of care (Gilmour and Brannelly 2010). A recent global movement that originated in Japan, Dementia Friendly Communities (Miyamoto, George and Whitehouse 2011), encourages that all environments are responsive to the needs of people with dementia, through for example, awareness raising about the effects of dementia on those affected by it, and developing knowledge about how people with dementia may be helped when they face difficulties.

It is expected that staff in specialist dementia homes have adequate training for positive person work with people with dementia, but the implementation of this is not always the case. In homes that are not 'dementia-specific', despite many people with dementia being present, there is likely to be little training for staff who lack knowledge about dementia, resulting in difficulty interacting well and understanding how to facilitate good care.

This small research project was part of a broader partnership between the unit staff and university staff to transition the unit from a clinical model of care to a more inclusive model. The clinical model was characterised by an emphasis on measuring health and bodily functions, with a focus on the satisfactory completion of tasks and procedures. New leadership led to the development of a 
philosophy recognising residents' needs to be actively involved in unit life. An important aspect of the vision was the aspiration that residents should live an ordinary life within a secure setting.

\section{Adopting a more inclusive approach with people with dementia and their families}

The revised inclusive care approach developed in an organic manner; it did not follow a previously developed template of principles characterised by programmes such as The Eden Alternative (Thomas, 2003). The culture change was produced through the synthesis of the ideas, energy and talents of the staff, based on the concepts of dignity, autonomy and humanity, understood as ways of maximising the abilities and experiences of people with dementia with their families. Dignity was as experienced by the person with dementia and assessed through the reaction to the care provided. Veugelers (2011) suggests autonomy is "not isolated individuality but it is the way the person relates to the other" (p. 1). Autonomy was understood as shared between the resident and their family, facilitating decision making that considered the resident's prior wishes alongside family priorities. The concern was to develop a life together as a community underpinned by moral values with the focus on the inclusion of people with dementia. Such individualised approaches developed in a facility specific manner lead to increased staff satisfaction and increased resident interactions (Brownie \& Nancarrow, 2013).

Effective cultural change is pervasive and infectious. Leadership and staff engagement are required to encourage care that is humanising, that engages people and encourages and sustains positive relationships between people. Adequate resourcing and staff educational investment is necessary to attain the desired shift (Nolan et al., 2008). Specific requirements to develop a resident centred culture include a focus on staff satisfaction, feedback, resourcing, capacity building, incentives, and developing a continuous learning culture (Luxford, Safran, and Delbanco 2011). Similarly, Brownie and Nancarrow (2013) in a systematic review identified that person centred care is multifaceted and requires environmental enhancement, positive social interactions, leadership shifts, staff empowerment, keyworker continuity and resident centred philosophy of care. Leadership is also a 
key factor in the promotion of good relationships between family and staff, as well as having continuity of staff and a strong philosophy of care that values family and service user contributions (Brown Wilson, 2009).

The process of transition to a revised care approach reflected many of these requirements. Staffing was reviewed and the number of qualified staff was reduced, and support workers employed so that there were more people available to provide care. The rules of the old culture were discarded, and residents were given choice about daily personal activities, such as when to get up, go to bed, visit the toilet or have drinks and snacks. Visiting times were opened up so that relatives and friends could visit more easily and the environment was rearranged to provide some privacy for families visits. A key worker system was introduced so that the support workers had direct responsibility for key areas of care and contact with relatives. A new activities programme was introduced and residents were encouraged to engage in meaningful activity identified from biographical knowledge of each person at the home, sourced from their family members where possible. Senior support workers were given responsibilities for management and leadership during their shifts, and all staff were involved in 'huddles' at the start of each shift to coordinate care and discuss resident's needs.

The engagement of residents' family members and friends was vital in developing the new vision and culture in the dementia unit. Austin et al. (2009) identified that families needed to be invited in to residential care home placements and given a role in care. Kemp et al. (2009) identified that a partnership approach to the care of people in residential care enabled better relationships between family members with family members accessed as a resource in their knowledge about the caregiving. Initially, the unit leader met with all family members individually to explain the issues with the clinical model of care and the desire to develop a more inclusive model. Building trust was a pivotal aspect of these meetings through honest and sincere communication about the desire to improve care. Engagement was maintained through three monthly group meetings with family and a newsletter. 
There was also investment in developing the learning culture of the unit. Research staff enriched the educational resources available through a programme of education suggested by staff that included best practice in communication; working with people who were distressed; family centred care; care of self and caring boundaries, and the unit as a community including the concepts of leadership and management. The focus of the education programme was to encourage the support workers to reflect on their roles and share the difficulties and positive experiences they encountered in providing care to people with dementia and their families.

\section{The Research}

The aim of this small research project was to explore the perceptions of care support staff and family members about the impact of the implementation of an inclusive model. The research design used a qualitative approach to explore the participants' perceptions and experiences, and was approved by the Massey University Human Ethics Committee (14/42). Staff and family focus groups were advertised through the unit communication channels and an information sheet provided to those interested about the research study, with focus groups organised by Massey University researchers.

Two focus groups were convened in September 2014 ten months after the implementation of the new model of care, one with family members and one with staff members who implemented the changes. The family focus group was held at $7 \mathrm{pm}$ in a slot that is usually reserved for a family information evening and lasted just over one hour, and eleven family members attended. Partners and adult children of residents participated. Five of the families had relatives who had been in the unit for less than one year, there was one family member who had contact with the unit for several years, and one who was very new to the unit. The family interview questions focused on their experiences of their relative's care including the activities they had been involved with and if they had noticed any changes or had staff commented on any changes in their relatives since the implementation of the new approach. 
The staff focus group was held in the afternoon and was led by an external facilitator as the researchers had been working directly with unit staff providing the education sessions. Nine staff members attended and the group ran for just over one hour. The staff members were all care workers who had been at the unit for between four months and eleven years. Those who had been at the unit for a longer time commented on the changes as they related to previous ways of working. Staff were asked to comment on the positive and challenging aspects of the approach, and impacts on residents and families. The focus group interviews were audio recorded and transcribed.

At the focus group interviews, family members were reminded of the inclusive approach adopted by the unit and asked to comment on the wellbeing of their family prompted using the Bradford Wellbeing Profile if necessary, (Bradford Dementia Group, 2008), their occupational activities and the staff approach. The staff participants were asked to comment on their experience of implementing the new way of working and the relationships with people with dementia and their families.

The focus group data was analysed using thematic analysis. Braun and Clarke's (2006) six stages of data analysis were followed, and involved data immersion through reading and re-reading the focus group transcripts, the identification of significant statements, followed by development into codes and themes. The analysis of the interview texts was led by one author, a second author read the interview texts and validated the identified themes. Interviews were recorded and transcribed, and one author generated the initial codes. Two of the authors explored the themes and reviewed them. Analysed data were discussed with the broader team, and two of the authors produced a project report.

\section{Experiences of care from staff and family perspectives}

At the focus groups, family members shared details about the care that their relative received, and this general conversation was greatly appreciated among the group, who commented that they 
were usually unable to share their experiences in this way. The care staff were initially reticent about sharing their experiences, but the conversation flowed as the group progressed. The themes personalised care for people with dementia, family involvement - continuing to care and staff competence and confidence to care were prominent in both focus groups.

\section{Personalised care for people with dementia}

The family participants talked about how personalised flexible care provided a less stressed and tense environment, unnecessary conflict and decreasing distressed behaviour were avoided by losing rigid 'rules'. The unit was calmer and more welcoming.

A good example of what people have been talking about in terms of getting to know residents individually - is that dad used to be very self-sufficient as in looking after himself, getting dressed and whatever it is. He's not now. He needs a bit more help in the morning. But he's also a very independently minded person and what the feedback both from him and from staff is that they have managed to find a way of getting alongside him and giving him support in the morning with his showering and his shaving and whatever, in a way that he accepts and feels good about... So I take that as quite a strong signal of the kind of philosophy I guess that people have been talking about. (Family focus group)

The shift from completing tasks to an emphasis on the experience of care by the person with dementia and the care giver humanised the care process for both.

I notice a lot people have got a - they are not really after the care, like if they are not in the mood we don't do care... later on when they are in good mood we do care, so it's not really all about their care...It is about being focussed on them. (Staff focus group)

The staff discussed changes in wellbeing for the people with dementia, such as accepting care more readily, or being more open and approachable. 
I know they got dementia and all that, but they can, they can trust us now. When it comes to care the women say you know, let's go and get freshened up for the day. Your family might visit love. They come with us straight away. Before they used to pull up a fist for example, say you go away or l'll punch your lights out, yeah. (Staff focus group)

One aspect of the less stressed environment, was that the staff were viewed as helpful and willing to find solutions to problems they encountered, using initiative and available resources. One family member complemented a staff member for his creativity when a person with dementia was admitted whose first language was not English. A translation 'app' was used to aid communication. Another family member talked about how synergistic relationships between staff members and people with dementia were recognised and nurtured so that people who got along well together were encouraged to spend time together. These relationships were fostered using keyworker roles so that the relationship had time to develop and grow.

And I think I'd probably just add to that that the personal care framework, that's a change that's come in since we were there because it's quite recent. But the idea of having one caregiver who's - and I see the picture up on his wall in his room, saying this is your special caregiver. That also has been a sign to us that they're really trying to figure out the person, match the personality. (Family focus group)

Staff participants noted the extent of the change in the atmosphere of the home. The staff members who participated in the focus groups had been there for varying lengths of time, some for many years. Those with longer histories reflected on the newer ways of working in contrast to previous ways of working.

I started four years ago, so for me the big change is really awesome to see. It was very clinical back in the day then. It is now a more homely environment. It's free-flowing and the people are more - family-wise they're more forthcoming, especially to the carers...so for me 
it's a good environment at the moment and we do flow well, from the AM (morning) right up to the nightshift. It just seems to all be flowing now and there's just a good sense of belonging here. (Staff focus group)

The staff focus group discussed the need for flexibility in providing activities based on what the residents wanted to do on a daily basis, rather than a roster of activities that maybe were not so appealing. Families were involved in providing information to the keyworker about what the person with dementia previously enjoyed so that activity or an adaptation of that activity may be offered. New activities were offered to see if the residents would like to try them.

He was sitting by himself so I gave a story about a soldier in the army as I knew that about him, so I went there and I said [name], would you like to read this book? So I face the book to him and he said oh, thank you very much, I would like to read that. (Staff focus group) Families also acknowledged the difficulties of providing meaningful activities, and the adaptation to the limitations imposed by dementia that may be experienced.

My husband is not a joiner-inner; he is at the top, giving orders or he's not there. [Laughter] And they are gradually getting him to go out and play bowls. He might only bowl the ball three times, but he's doing it and he's actually participating in some of the exercise programs today. He doesn't think much of them you know. He'd much rather be running track and winning, winning a race, you know? But they don't really know much what they're doing, but he's doing it. [Laughs] So he's no longer just one of the others; he is definitely an individual. (Family focus group).

These attempts at personalised care were seen as beneficial by the family members and staff. Lightening the atmosphere and removing unnecessary rules that had benefitted the organisation rather than the residents brought about a change in the relations between people. 
Although family involvement is an aim in residential care for people with dementia, family members often feel as though they are excluded from decisions regarding their family members, or from information that staff refrain from disclosing. Getting the right level of family involvement is dependent on knowing what level of involvement family members want, and how they like to be informed, and depends on staff making sure that family are included. The family members who attended the focus group felt very welcome at the unit whenever they visited, and felt cared for by the staff who recognised when they experienced distress during their visit.

From the time you come in, whoever's on reception is very friendly and one day it was really, really bad. And one of the staff let me out and came out with me and gave me a hug and said look, I don't know a lot yet, I'm quite new here, but any time you need to talk just contact me. (Family focus group)

The family members at the focus group agreed that they were given enough information and requests for involvement to shape the care provided to their relative. They described how members of staff had called them when an incident had occurred. When family members were only able to visit at weekends, contact with the keyworker was difficult to maintain, but staff on duty gave adequate progress reports.

Family members were not always aware that the feedback provided was 'edited' to some degree such as when the staff faced difficulty with providing personal care.

One thing I was surprised by, like they you know, they give me all the feedback that I need to know. I actually didn't realise how badly my mother was behaving sometimes and they, they hadn't told me that. And I went in one morning. I don't normally go in the morning and the caregiver, a caregiver was trying to get her dressed and my mother was punching and punching her and swearing at her. (Family focus group) 
When the family member talked about this in the focus group, others empathised with their situation, and this discussion led to recognition of the skills and patience of the staff. In the staff focus group the loosening of the management responsibility so that staff members were able to communicate with families (previously only nurses had been allowed to talk to family members) led to increased role satisfaction.

It's a good sense of - it's a feeling of being valued, valued as a care worker, valued as a carer and most of all being able to communicate on a level with the family, that you know you're on an understanding with them and they understand you, yeah. (Staff focus group)

And so it's really good having that one on one communication with families, like maybe we we never did have that when we first started here. It was very cold. It was very clinical. (Staff focus group)

The changes in the home had opened communication channels between families and staff members. Rather than being afraid of giving the 'wrong' answer, staff were encouraged to discuss care needs and get advice from families about how best to approach their family members.

\section{Competence and confidence to care}

In the staff focus group competence and confidence to care well was discussed. Competence was about knowing why they were taking particular approaches with residents, having the knowledge to care well. Staff in the focus group discussed the recent training that was available to them where they had requested more information about the impact of dementia, and how to respond well to avoid distress.

Oh well to me it's working for me because it's giving me more experience of how to, to provide high care, high standard of care and how to meet their needs. (Staff focus group) 
Confidence was about being strong enough to accept feedback about their caring abilities including suggestions for improvements. The keyworker role meant that the support workers were able to provide specific attention to the people with dementia and getting to know them meant that they were able to grow in confidence about how to interact well with them by spending more time with the resident. Staff described a sense of belonging and responsibility with the role and took positive feedback from the residents as indicators of doing good work.

The keyworker role had been part of a broader institutional change that had seen a policy change so that the staff were dedicated to the unit, rather than available to work anywhere across the campus (including hospice care). Being able to have a dedicated role on the unit and not be moved to other roles or duties within the organisation enabled the staff to fulfil their roles. Senior staff made sure that good work was recognised and that people were thanked for their efforts.

Family members described the characteristics of the staff as caring, a team that supported each other, gentle in their approach to people, affectionate, engaged, learning and with an enabling approach.

Another thing I've really noticed since the change is how the staff, no matter how young or new they are, are continually just looking round the room. If someone seems upset or stuck and they're trying to stand up, there's someone there and they're working together. (Family focus group)

Family members commented on different levels of experience, skills and abilities in helping people with dementia.

Some of the staff are older, some are younger and they've all got to learn, you know? And some residents can be more difficult than others and I know that mine can be a bit resistant 
to a few things. So you know, some staff can handle that but it's a bit of a learning curve for some of them as well. (Family focus group)

One aspect that was prevalent in the staff focus group was that staff reported that under their new leadership they felt more confident in their work, a marked difference to not knowing how people were going to react to them trying new things with the residents or talking to resident's families. These clear boundaries built competence and allowed the care staff to experiment and be creative in the care they provided, which was in turn appreciated by the family members.

\section{Achieving an ordinary life}

In this discussion, the ordinariness that was the aim of the new model of care is considered in light of the literature focused on the importance of personalised care for people with dementia, family involvement and staff competence to care. Feeling 'at home' is a synergy of the material environment, the community of people and the quality of the relationships within that environment whilst being valued as a unique person. Morgan and Stewart (1997) identified that social environments were more important that physical environments for the wellbeing of people with dementia. Edvardsson et al (2010) researched the meaning of person-centeredness to people with dementia who reported the continuation of the self, preservation of identity and living a 'normal' life central to their interpretation of person-centeredness. Brownie and Nancarrow (2013) identified person centred care as multifaceted, including environmental enhancement, social interactions, leadership, staff empowerment and a keyworker approach for continuity. Implementation of psychosocial measures include connecting with people, meaningful contributions, seeing beyond the illness, awareness of different approaches to care and that psychosocial measures bring specific outcomes. These outcomes are dependent on family involvement, flexibility, resources, institutional philosophy and attitudes of staff (Lawrence, Fossey, Ballard, Moniz-Cook, \& Murray, 2012). Leadership is crucial in the implementation and sustainability of psychosocial approaches (Helgesen, Larsson, \& Athlin, 2010). 
The staff reflections highlighted their feeling of being valued for their contributions through their increased interactions with families. Good communication and clear expectations about communication strategies helps the process of establishing and maintaining relationships between care staff and family members. Austin et al. (2009) identified that families needed to be invited in to residential care home placements and given a role in care. This may be achieved through staff/family collaborations on a number of levels. Rotation of staff off the unit was to be avoided so that families knew who to expect, so that contact and trust could be established.

Cooperative communication based on conflict resolution supports better relationships between families, staff and residents (Robison et al., 2007), and aids collaboration and partnerships in care (Pashby, Hann, and Sunico 2009). Kemp et al (2009) advocate a partnership approach to enable family members to be accessed as a resource in caregiving. Bramble, Moyle and McAllister (2009) identify that improving family - staff relationships has the potential to reduce conflict and improve the long-term care environment, relieve the pressure of work overload, decrease staff frustration and reduce negative reactions to family caregivers.

Brownie and Nancarrow (2013) in their systematic review of patient-centred care, noted that interventions influence staff satisfaction and the ability to provide care focused on the individual needs. In this study the focus on organisational change to effect better care included more training and staff reconfiguration to provide more hands-on availability and frequent activities to engage the people with dementia and be with them and their families. Education, age, work experience, care unit size, and specialised training are all associated with staff attitudes and that encouraging further training and development helps to improve attitudes (Kada, Nygaard, Mukesh, \& Geitung, 2009). The unit achieved a significant shift in the care for people with dementia who accepted care because they trusted the people who were providing it and this was substantiated by the people they trusted - their families. 
A limitation of this research is that the people with dementia at the unit were not involved directly in data collection. An extension of this project would be to spend time on the unit with people with dementia to record their experiences of the care environment and interview them about the care they receive. Adequate resources were not available to enable this design, as the research was unfunded. The researchers were mindful of the time commitment required for this research approach, and although this was desirable it was not possible. Involving people with dementia would have given a clearer picture of the experience of care, and only they who receive care are able to comment on whether the care provided met their needs. We were pleased to have the voice of families who were able to comment on their experiences and continuing caregiving roles.

\section{Conclusion}

The process of introducing a new culture of care focused on maximising ability and providing warm and friendly social interaction in order to promote wellbeing and the possibility of living an ordinary life. The implementation of the model was supported through group activity programmes, changes in the physical environment and the staffing configuration, increased opportunities for family participation, attention to family centred care planning and documentation and a staff driven education programme. The research findings identified positive ways of working that benefitted staff and families who reported increased wellbeing for the people with dementia on the unit. The development of a well-articulated and systematically implemented local care model provided opportunities for family and staff creativity and participation along with staff capacity building. 
Acknowledgement

Thanks to the staff and family members who gave their time and expertise to the research. A special thanks to Rachel Clarke who completed the literature search for the project and conducted one of the focus groups. This project was successful in harnessing the goodwill and abilities of staff from two institutions with the ultimate aim of sustaining a new culture of care in a specialist unit for people with dementia. The partnership was informal and relied on reciprocal activities to generate contributions in time and expertise from both partner organisations. 


\section{References}

Austin. W., Goble, E., Strang, V., Mitchell, A., Thompson, E., Lantz, H., Balt, L., Lemermeyer, G., \& Vass, K. (2009). Supporting relationships between family and staff in continuing care settings. Journal of Family Nursing, 15(1), 360-383. doi: 10.1177/1074840709339781

Bradford Dementia Group (2008) The Bradford Well-being Profile, Bradford: University of Bradford.

Bramble, M., Moyle, W., \& MacAllister, M. (2009). Seeking connection: Family care experiences following long term dementia care placement. Journal of Clinical Nursing, 18(22), 3118 3125. doi:10.1111/j.1365-2702.2009.02878.x.

Braun, V., \& Clarke, V. (2006). Using thematic analysis in psychology. Qualitative Research in Psychology, 3(2), 77-101. doi:10.1191/1478088706qp063oa

Brownie, S., \& Nancarrow, S. (2013). Effects of person centred care on residents and staff in aged care facilities: A systematic review. Clinical Interventions in Aging, 8, 1- 10. doi:10.2147/CIA.S38589

Brown Wilson, C. (2009). Developing community in care homes through a relationship centred approach. Health and Social Care in the Community, 17(2), 177 - 186. doi:10.1111/j.13652524.2008.00815.x.

Edvardsson, D., Fetherstonhaugh, D., \& Nay, R. (2010). Promoting a continuation of self and normality: Person-centred care as described by people with dementia, their family members and aged care staff. Journal of Clinical Nursing, 19(17-18), 2611 - 2618. doi:10.1111/j.13652702.2009.03143.x

Gilmour, J.A., \& Brannelly, T. (2010). Representations of people with dementia - subaltern, person, citizen. Nursing Inquiry, 17(3), 240-247. doi:10.1111/j.1440-1800.2009.00475.x

Helgesen, A. K., Larsson, M., \& Athlin, E. (2010), 'Patient participation' in everyday activities in special care units for persons with dementia in Norwegian nursing homes. International Journal of Older People Nursing, 5(2), 169 - 178. doi:10.1111/j.1748-3743.2010.00223.x

Kada, S., Nygaard, H.A., Mukesh, B.N., \& Geitung, J.T, (2009). Staff attitudes towards institutionalised dementia residents. Journal of Clinical Nursing, 18(16), 2383 - 2392. doi:10.1111/j.13652702.2009.02791.x

Kemp,C., Ball, M., Perkins, M., Hollingsworth, C., \& Lepore, M. (2009). "I get along with most of them": Direct care workers relationships with resident's families in assisted living. The Gerontologist, 49(2), 224-235. doi:10.1093/geront/gnp025

Lawrence, V., Fossey, J., Ballard, C., Moniz-Cook, E., \& Murray, J. (2012). Improving quality of life for people with dementia in care homes: Making psychosocial interventions work. British Journal of Psychiatry, 201(5), 344 - 351. doi: 10.1192/bjp.bp.111.101402

Luxford, K., Safran, D., \& Delbanco, T. (2011). Promoting patient-centered care: A qualitative study of facilitators and barriers in healthcare organizations with a reputation for improving the patient experience. International Journal of Quality in Health Care,23(5), 510-515. doi:10.1093/intqhc/mzr024 
Miyamoto M, George DR, Whitehouse PJ (2011), Government, professional and public efforts in Japan to change the designation of dementia (chiho), Dementia, 10(4) 475-486, DOI: $10.1177 / 1471301211416616$

Morgan, D.G., \& Stewart, N.J. (1999). The physical environment of special care units: Needs of residents with dementia from the perspective of staff and caregivers. Qualitative Health Research, 9(1), 105-118.

Nolan,M., Davies, S., Brown, J., Wilkinson, A., Warnes, T., McKee, K., Flannery,J., \& Stasi, K. (2008). The role of education and training in achieving change in care homes: A literature review . Journal of Research in Nursing, 13(5), 411-433, doi:10.1177/1744987108095162

Savva, G., Zaccai,J., Matthews, F., Davidson, J.,McKeith, I., Brayne, C., Medical Research Council Cognitive Function and Ageing Study. (2009). Prevalence, correlates and course of behavioural and psychological symptoms of dementia in the population. British Journal of Psychiatry, 194,212-219. doi:10.1192/bjp.bp.108.049619

Swaffer, K. (2015). Dementia and prescribed dis-engagement. Dementia, 14(1), 3-6. doi:10.1177/14713012145481

Pashby, P., Hann, J., \& Sunico, M.E.S. (2009). Dementia care planning: Shared experience and collaboration. Journal of Gerontological Social Work, 52(8), 837 - 848. doi:10.1080/01634370903088051

Robison, J., Curry, L., Gruman, C., Porter, M., Henderson, C.R., \& Pillemer, K. (2007). Partners in caregiving in a special care environment: Cooperative communication between staff and families on dementia units. The Gerontologist, 47(4), 504-515.

Thomas, W. (2003). Evolution of Eden. In A. Weiner \& Ronch, J. (eds.) Cultural change in long-term care (pp.141-157). New York, NY: The Haworth Social Work Practice Press.

Veugelers, W. (2011). Introduction: Linking autonomy and humanity. In W. Veugelars (Ed.). Education and humanism: Linking autonomy and humanity (pp. 1-7). Rotterdam, Netherlands: Sense Publishers.

Vladeck, B. (2003). Unloving care revisited: The persistence of culture. In A. Weiner \& Ronch, J.(Eds.). Cultural change in long-term care (pp.1-9). New York, NY: The Haworth Social Work Practice Press. 